\title{
Development of Epidural and Paraspinal Abscesses after Insufficient Evaluation and Treatment of Acute Pyelonephritis Caused by Staphylococcus aureus
}

\author{
Mi Jeoung Kim ${ }^{1, *}$, Hyang Mo Koo ${ }^{1}$, Woo Joo Lee ${ }^{2}$, Jin Hwan $\mathrm{Choi}^{3}$, Mi Nyong Choi ${ }^{4}$, Sang Young Park \\ Woo Jung Kim ${ }^{5}$, Seung Yeon Son ${ }^{6}$ \\ 'Division of Nephrology, Department of Internal Medicine, Inseong Hallym Hospital, Incheon, Korea \\ ${ }^{2}$ Division of Infectious Diseases, Department of Internal Medicine, Inseong Hallym Hospital, Incheon, Korea \\ ${ }^{3}$ Department of Neurosurgery, Inseong Hallym Hospital, Incheon, Korea \\ ${ }^{4}$ Department of Radiology, Inseong Hallym Hospital, Incheon, Korea \\ ${ }^{5}$ Department of Internal Medicine, Inseong Hallym Hospital, Incheon, Korea \\ ${ }^{6}$ Division of Nephrology, Department of Internal Medicine, Gimpo Woori Hospital, Gimpo, Korea
}

Diagnoses of pyelonephritis caused by Staphylococcus aureus should be accompanied by investigations of concomitant bladder obstruction and metastatic infections, especially to the spine or heart. Complicated pyelonephritis due to $S$. aureus requires more than 2 weeks of antibiotics, which is the typically recommended treatment duration for pyelonephritis. We describe a patient who was diagnosed with complicated epidural and paraspinal abscesses after insufficient evaluation and treatment of acute pyelonephritis due to $S$. aureus. A 62-year-old man with type 2 diabetes was admitted with fever, increased urinary frequency, and left flank pain. He was diagnosed with acute pyelonephritis caused by $S$. aureus. His fever and flank pain subsided after 3 days of intravenous antibiotics. Evaluation of bladder obstruction and metastatic infection were not performed, as he declined further evaluation. The patient was discharged with oral antibiotics and was requested to attend weekly appointments but was lost to follow-up. One month later, the patient presented at the outpatient clinic with similar symptoms. Computed tomography showed recurrent pyelonephritis and a distended bladder. His flank pain persisted despite administration of an opioid agent. Therefore, magnetic resonance imaging was performed, revealing epidural and paraspinal abscesses. Ultrasound-guided aspiration of the paraspinal muscle layer was performed, and blood and percutaneous aspirated fluid cultures revealed $S$. aureus growth. The pattern of antimicrobial sensitivity was identical to that at his first admission. Following more than 4 weeks of antibiotics, magnetic resonance imaging showed the abscesses had decreased in size. The patient was discharged without neurologic sequelae and was provided with oral antibiotics.

Keywords: Staphylococcus aureus; Pyelonephritis; Epidural Abscess; Urinary Tract Infections 


\section{INTRODUCTION}

Acute pyelonephritis demonstrates a high annual incidence of 35.7 cases per 10,000 people. This rate is increasing owing to greater numbers of elderly, immunocompromised patients. ${ }^{1)}$ In uncomplicated pyelonephritis cases, appropriate antibiotics improve fever within 72 hours and reduce leukocytosis and elevated C-reactive protein (CRP) levels. Uncomplicated pyelonephritis generally requires 7-14 days of antibiotic therapy. ${ }^{2,3)}$ However, when Staphylococcus aureus is the pathogen, dissemination can occur, and a prolonged duration of antibiotic treatment is required. ${ }^{4)}$

$S$. aureus rarely causes pyelonephritis in the general population. In $1997, S$. aureus accounted for only $0.5 \%$ and $1.3 \%$ of isolates obtained from urine in general populations from Great Britain and France, respectively. ${ }^{5)}$ In South Korea, $S$. aureus is classified as a rare cause of community-acquired pyelonephritis. ${ }^{3)}$

We describe a patient who was diagnosed with complicated epidural and paraspinal abscesses after insufficient evaluation and treatment of acute pyelonephritis due to $S$. aureus.

\section{CASE REPORT}

A 62-year-old man was admitted with a 5-day history of fever, increased urinary frequency, and left flank pain. He had no specific medical or surgical history, except for a 5-year history of type 2 diabetes treated with insulin and oral hypoglycemic agents. Physical examination revealed a blood pressure of $120 / 70 \mathrm{~mm} \mathrm{Hg}$, temperature of $38.4^{\circ} \mathrm{C}$, heart rate of 76 beats/min, and respiration rate of 20 breaths/ min. His breathing sound was clear, and his heartbeat was regular without murmurs. However, knocking tenderness in his left flank area was noted. The initial laboratory data revealed a white blood cell (WBC) count of $14,680 / \mathrm{mm}^{3}$, hemoglobin level of $12.2 \mathrm{~g} / \mathrm{dL}$, platelet count of $373,000 / \mathrm{mm}^{3}$, CRP level of $>12 \mathrm{mg} / \mathrm{dL}$, and hemoglobin Alc level of $12.5 \%$, indicating that his diabetes was poorly controlled. Blood urea nitrogen, serum creatinine, alanine transaminase, and aspartate transaminase levels were within the normal range. Urinalysis showed white and red blood cell counts of 3-5/high-power field (HPF) and 1015/HPF, respectively. Contrast-enhanced abdominal computed tomography (CT) scans showed a multifocal decreased perfusion defect of the left kidney. Blood and urine culture samples were obtained, and intravenous ceftriaxone ( $2 \mathrm{~g} / \mathrm{d}$ ) was administered immediately. His fever subsided within 72 hours, and laboratory data demonstrated improvement. Five days after admission, the initial urine and blood cultures revealed growth of methicillin-sensitive $S$. aureus (MSSA). Urologic evaluation regarding bladder obstruction and screening for metastatic infection were recommended. However, the patient declined further evaluation, as his discomfort had subsided, and his poor socioeconomic status impeded the payment of additional medical costs. Eight days after admission, laboratory data demonstrated further im-

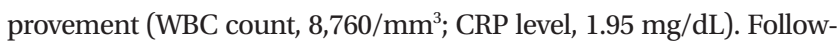
up urine and blood cultures obtained on day 5 revealed no bacterial growth. The patient was therefore discharged with oral ciprofloxacin (500 mg every 12 hours) for 7 days. He was asked to attend the outpatient clinic for 1 month to receive maintenance ciprofloxacin, but he failed to attend.

Approximately 1 month later, the patient presented with a 7-day history of symptoms consistent with those at his initial visit. His vital signs were as follows: a blood pressure of $110 / 70 \mathrm{~mm} \mathrm{Hg}$, temperature of $38.4^{\circ} \mathrm{C}$, heart rate of 115 beats/min, and respiration rate of 20 breaths/ min. Bilateral costovertebral angle tenderness was noted. Laboratory data revealed a WBC count of 15,870/ $\mathrm{mm}^{3}$, hemoglobin level of $9.6 \mathrm{~g} /$ $\mathrm{dL}$, platelet count of $375,000 / \mathrm{mm}^{3}$, and CRP level of $>12 \mathrm{mg} / \mathrm{dL}$. Blood urea nitrogen, serum creatinine, alanine transaminase, and aspartate transaminase levels were within the normal range. Urinalysis showed a WBC count of 30-50/HPF with many bacteria and a red blood cell count of 5-10/HPF. A CT scan showed a multifocal decreased perfusion defect of both kidneys (Figure 1) with bladder distension. Urologic evaluation demonstrated a residual urine volume of $250 \mathrm{~mL}$. Prostatespecific antigen levels were normal. He was therefore diagnosed with recurrent pyelonephritis and neurogenic bladder. An oral alpha blocking agent and intravenous ciprofloxacin (400 mg every 12 hours) were administered. Two days after readmission, his left lower flank pain persisted, despite a fentanyl patch $(12 \mu \mathrm{g} / \mathrm{h})$. Although there were no neurologic deficits, a neurosurgeon was consulted regarding his severe pain and the possibility of concomitant spinal disease. Magnetic resonance imaging (MRI) with gadolinium enhancement was performed, revealing epidural abscesses at L4-5 and paraspinal abscesses of the muscles at L3-S2 (Figure 2). Ultrasound-guided aspiration of the paraspinal muscle layer was performed using an 18-gauge needle, and approximately $10 \mathrm{~mL}$ of yellow-white mucoid fluid was aspirated. The epidural abscesses were not drained, as there were no neurologic deficits. Cultures of blood and drained fluid revealed MSSA growth (Table 1) with antimicrobial sensitivity identical to that of the initial culture. Urine cultures revealed growth of $<1,000$ colony forming units $/ \mathrm{mL}$, al-

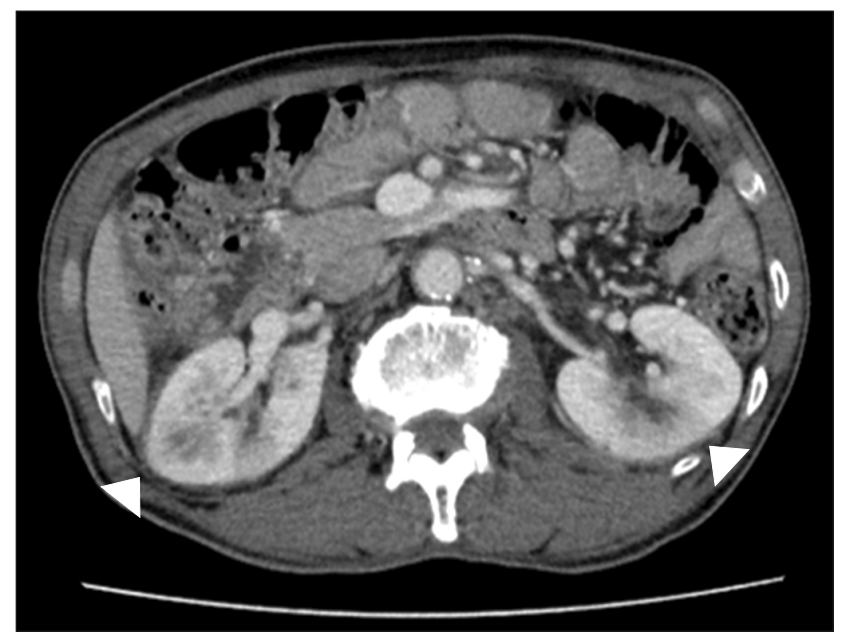

Figure 1. A dynamic contrast-enhanced computed tomography image of the kidney showing bilateral acute pyelonephritis. A delayed-phase image showed multifocal patchy areas (arrowheads) of low attenuation in both kidneys. 


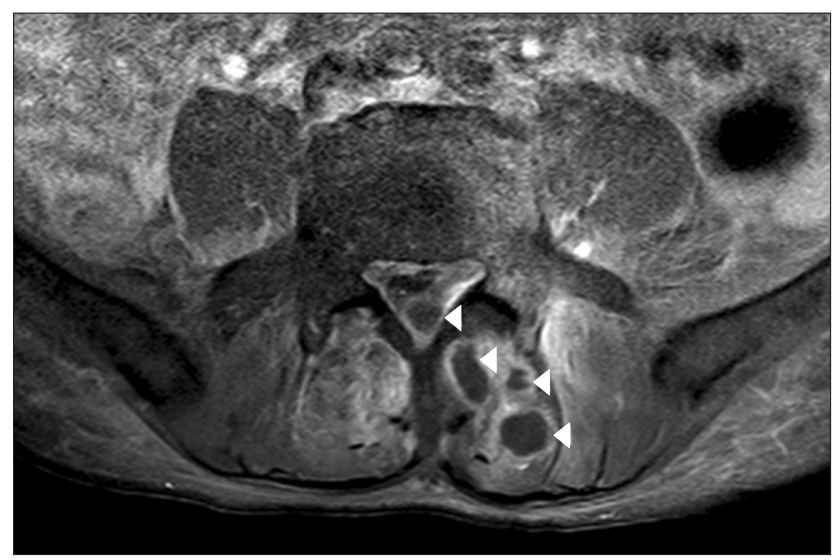

Figure 2. Magnetic resonance image of the lumbar spine shows epidural and paraspinal abscesses (arrowheads). Axial contrast-enhanced T1-weighted images of the L5 show a low signal intensity lesion in the left dorsal epidural space with peripheral rim enhancement. Similar lesions can be seen in the left paraspinal muscles, indicating epidural and paraspinal abscesses.

Table 1. Antibiotic susceptibility of Staphylococcus aureus

\begin{tabular}{ll}
\hline \multicolumn{1}{c}{ Antibiotic } & Sensitivity (minimum inhibitory concentration) \\
\hline Ciprofloxacin & $\mathrm{S}(1)$ \\
Clindamycin & $\mathrm{S}(\leq 0.25)$ \\
Erythromycin & $\mathrm{S}(\leq 0.25)$ \\
Fusidic acid & $\mathrm{S}(\leq 0.5)$ \\
Gentamicin & $\mathrm{S}(\leq 0.5)$ \\
Habekacin & $\mathrm{S}(\leq 1)$ \\
Linezolid & $\mathrm{S}(1)$ \\
Mupirocin & $\mathrm{S}(\leq 2)$ \\
Nitrofurantoin & $\mathrm{S}(\leq 16)$ \\
Oxacillin & $\mathrm{S}(\leq 0.25)$ \\
Quinupristin/dalfopristin & $\mathrm{S}(\leq 0.25)$ \\
Rifampin & $\mathrm{S}(\leq 0.5)$ \\
Teicoplanin & $\mathrm{S}(\leq 0.5)$ \\
Telithromycin & $\mathrm{S}(\leq 0.25)$ \\
Tetracycline & $\mathrm{S}(\leq 1)$ \\
Tigecycline & $\mathrm{S}(\leq 0.12)$ \\
Trimethoprim/sulfamethoxazole & $\mathrm{S}(\leq 10)$ \\
Vancomycin & $\mathrm{S}(1)$ \\
Penicillin G & $\mathrm{R}(0.25)$ \\
\hline
\end{tabular}

$\mathrm{S}$, sensitive; R, resistant.

though the bacterial strain could not be identified. Transesophageal echocardiography revealed no evidence of infective endocarditis. Nafcillin was injected every 4 hours for 22 days. However, neutropenia occurred, and nafcillin was replaced with vancomycin for an additional 30 days. On day 57 after readmission, MRI showed the abscesses had decreased in size (Figure 3). Residual urine volume was $40 \mathrm{~mL}$, and no discomfort was observed. Laboratory data were nearly within the normal range (WBC count, 6,490/ $\mathrm{mm}^{3}$; CRP level, $0.35 \mathrm{mg} / \mathrm{dL}$ ). On day 59 , he was discharged without neurologic sequelae and was prescribed ciprofloxacin (500 mg every 12 hours) until a repeat MRI showed no remnant abscesses.

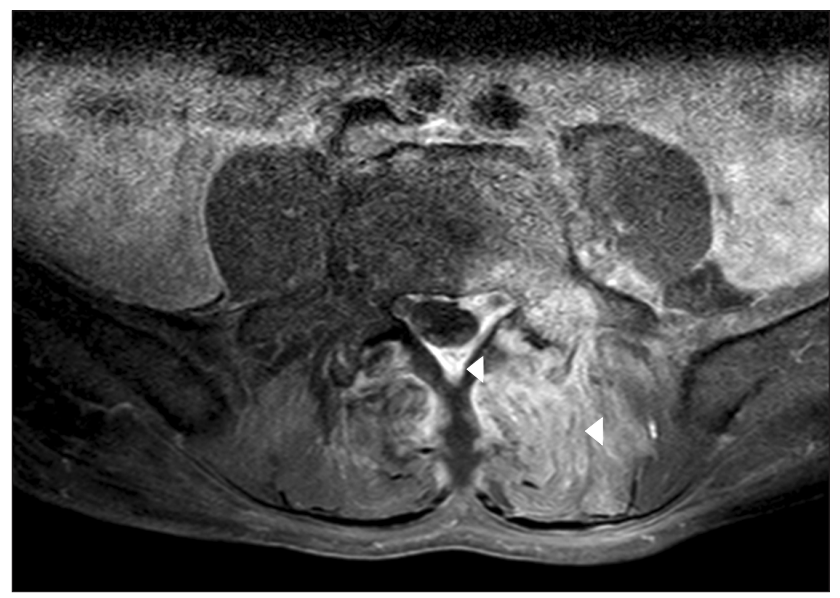

Figure 3. Follow-up magnetic resonance images showing that the epidural and paraspinal abscesses (arrowheads) had decreased in size. Axial contrast-enhanced T1-weighted images of the L5 still showed ill-defined heterogeneous enhancement of the paraspinal muscles, representing a phlegmon or granulation tissue. However, abscesses of the dorsal epidural and paraspinal muscles were no longer visible.

\section{DISCUSSION}

According to the Infectious Diseases Society of America guidelines, uncomplicated $S$. aureus bacteremia (SAB) is defined by five factors: the exclusion of endocarditis, no implanted prostheses, $S$. aureus-negative blood cultures 2-4 days after initial presentation, defervescence within 72 hours of therapy, and no evidence of metastatic infection sites. These guidelines suggest administering antibiotic treatment for a minimum of 2 weeks for uncomplicated SAB, and a minimum of 4 weeks for complicated SAB, personalized to the patient's condition. ${ }^{4)}$

$S$. aureus can disseminate, and it is therefore difficult to differentiate primary pyelonephritis caused by $S$. aureus from secondary $S$. aureus bacteriuria (SABU). ${ }^{5,6)}$ There is controversy regarding the appropriate diagnostic tests required to exclude occult foci caused by SAB. Consequently, it is difficult to determine the minimum duration of antibiotic treatment required for $\mathrm{SAB}^{4}{ }^{4}$

In 2009, Choi et al. ${ }^{6)}$ showed that patients with SAB and SABU had higher rates of urinary tract infection, bladder obstruction, and spinal infection compared with patients with SAB only. The association of SABU with spinal infection may result from the venous system acting as a route for propagation of infection between the pelvic organs and spinal region without systemic dissemination. ${ }^{7)}$

In agreement, our patient had concomitant pyelonephritis, neurogenic bladder, and epidural and paraspinal abscesses without infective endocarditis. On initial admission, the patient's fever and pain subsided within 72 hours of treatment, and laboratory data showed significant improvements. The patient declined additional examinations to assess bladder obstruction and metastatic infection, as his poor socioeconomic status impeded the payment of additional medical costs. Thus, it was difficult to persuade him of the importance of further examinations. However, his condition worsened after readmission, and he agreed to undergo the required examinations. Subsequently, neu- 
rogenic bladder and epidural and paraspinal abscesses were diagnosed.

Epidural abscess is rare, with a frequency of $0.2-2$ cases $/ 10,000$ hospital admissions and a mortality rate of $10 \%$ to $30 \%$ owing to potentially permanent neurological deficits. However, the incidence is increasing, owing to increased numbers of immunocompromised patients and advancements in diagnostic imaging techniques. Epidural abscesses characteristically have an insidious onset of symptoms, with the classic symptom triad being localized pain, fever, and neurological deficits. Overall, $74 \%, 64 \%$, and $10 \%-15 \%$ of patients reportedly present with pain, fever, and all three symptoms, respectively. Hence, the diagnosis of an epidural abscess is often missed on initial examination, and delays in treatment dramatically worsen prognosis owing to the risk of neurologic sequelae. Therefore, a high level of clinical suspicion is important for timely diagnosis and treatment. ${ }^{8)}$ As the patient in this case had both epidural and paraspinal abscesses, his pain was likely more severe than it would have been due to an epidural abscess alone, and therefore, the suspicion of spinal infection was relatively high. The current gold standard for the diagnosis of an epidural abscess is MRI with gadolinium contrast, and the most common causative pathogen is $S$. aureus. Infection with $S$. aureus can be managed successfully with conservative treatment comprising antibiotics and spinal bracing. Surgical intervention is indicated when neurologic deficits are present. ${ }^{9)}$

It is uncertain whether the abscesses were present initially, in which case they may have been partially treated and then worsened after the cessation of antibiotics. However, this case emphasizes the importance of investigating concomitant bladder obstruction and metastatic infection in patients diagnosed with community-acquired pyelonephritis due to $S$. aureus. The adequate treatment of bladder obstruction with antibiotics, despite improvements in patient symptoms and laboratory tests, should be carefully considered by the primary care physician.

\section{CONFLICT OF INTEREST}

No potential conflict of interest relevant to this article was reported.

\section{REFERENCES}

1. Bae MH, Park CH, Cho YS, Joo KJ, Kwon CH, Park HJ. Effects of diabetes mellitus and HbAlc on treatment prognosis in uncomplicated acute pyelonephritis. Korean J Urogenit Tract Infect Inflamm 2015;10: 41-8.

2. Bang SH, Chang IH, Han JH, Ahn SH. C-reactive protein is a useful marker to predict the severity and early response of acute pyelonephritis in women. Korean J Urol 2007;48:1143-8.

3. The Korean Society of Infectious Diseases; The Korean Society for Chemotherapy; Korean Association of Urogenital Tract Infection and Inflammation; The Korean Society of Clinical Microbiology. Clinical guideline for the diagnosis and treatment of urinary tract infections: asymptomatic bacteriuria, uncomplicated \& complicated urinary tract infections, bacterial prostatitis. Infect Chemother 2011;43:1-25.

4. Liu C, Bayer A, Cosgrove SE, Daum RS, Fridkin SK, Gorwitz RJ, et al. Clinical practice guidelines by the infectious diseases society of america for the treatment of methicillin-resistant Staphylococcus aureus infections in adults and children. Clin Infect Dis 2011;52:e18-55.

5. Muder RR, Brennen C, Rihs JD, Wagener MM, Obman A, Stout JE, et al. Isolation of Staphylococcus aureus from the urinary tract: association of isolation with symptomatic urinary tract infection and subsequent staphylococcal bacteremia. Clin Infect Dis 2006;42:46-50.

6. Choi SH, Lee SO, Choi JP, Lim SK, Chung JW, Choi SH, et al. The clinical significance of concurrent Staphylococcus aureus bacteriuria in patients with S. aureus bacteremia. J Infect 2009;59:37-41.

7. Pearce JM. The craniospinal venous system. Eur Neurol 2006;56:136-8.

8. Van Bergen J, Plazier M, Baets J, Simons PJ. An extensive spinal epidural abscess successfully treated conservatively. J Neurol Neurosurg Psychiatry 2009;80:351-3.

9. Butler JS, Shelly MJ, Timlin M, Powderly WG, O’Byrne JM. Nontuberculous pyogenic spinal infection in adults: a 12-year experience from a tertiary referral center. Spine (Phila Pa 1976) 2006;31:2695-700. 